\title{
COMMENTARY
}

\section{Assessment of fluid responsiveness during increased intra-abdominal pressure: keep the indices, but change the thresholds}

\author{
Benoit Tavernier* and Emmanuel Robin \\ See related research by Jacques et al., http://ccforum.com/content/15/1/R33
}

\begin{abstract}
Dynamic variables of fluid responsiveness are useful guides for fluid management in patients under controlled positive pressure ventilation. In the previous issue of Critical Care, Jacques and colleagues show that these variables remain reliable predictors of fluid responsiveness in a porcine model of intra-abdominal hypertension, but threshold values are higher than during normal intra-abdominal pressure. Their threshold values, however, cannot be applied to clinical practice. This study suggests that intra-abdominal pressure must be measured in critically ill patients, and 'supranormal'values of dynamic variables should be analyzed with caution. The 'fluid responsive part' of an increased dynamic variable in such patients may be estimated by measuring its change during a fluid challenge.
\end{abstract}

In the previous issue of Critical Care, Jacques and colleagues [1] report that respiratory variations in stroke volume (SVV) and in arterial pulse pressure (PPV) remain reliable indices of fluid responsiveness in a porcine model of intra-abdominal hypertension (IAH). Threshold values, however, are higher than during normal intra-abdominal pressure (IAP), so that a supranormal' SVV or PPV does not necessarily mean fluid responsiveness during IAH.

Why is this study important? Dynamic indices such as PPV and SVV are accurate predictors of volume responsiveness in critically ill patients under controlled positive pressure ventilation, with an accuracy greater than that of

*Correspondence: benoit.tavernier@chru-lille.fr

Pôle d'Anesthésie Réanimation, CHU de Lille, and EA 1046, Université Lille II, IMPRT-IFR 114, Lille, France traditional static indices of cardiac preload. The mean threshold values allowing optimal discrimination between fluid responders and non-responders were $12.5 \pm 1.6 \%$ for PPV and $11.6 \pm 1.9 \%$ for SVV in a recent systematic review of the literature [2]. These results, however, did not include patients where conditions prevented correct measurement of these indices (such as cardiac arrhythmias or spontaneous ventilation) or may have been associated with reduced accuracy (especially small tidal volumes or acute cor pulmonale). A third category of circumstances, where dynamic indices remain robust indicators of fluid responsiveness if (and only if) used with different thresholds, may also be identified and constitute an additional refinement in PPV/SVV interpretation. It was suspected early on that the magnitude of tidal volume would influence the threshold value of functional hemodynamics [3]. More recently, Biais and coworkers [4] showed that prone position (for scoliosis surgery) does not alter the ability of both PPV and SVV to predict fluid responsiveness, but induces a significant increase in PPV and SVV, probably related to a decrease in static compliance of the respiratory system. Accordingly, the 'optimal' threshold value for PPV in this study was $11 \%$ in the supine position, and $15 \%$ in the prone position [4]. In recent years, elevated IAP has been increasingly recognized in medical and surgical critically ill patients [5]. Cardiovascular dysfunction and failure are commonly encountered in the patient with IAH, and one of the bases of management is optimization of systemic perfusion and organ function, which includes accurate assessment of preload and preload responsiveness [5]. However, static indices of preload are difficult to interpret in patients with IAH [6]. Assessing fluid responsiveness with the passive leg raising maneuver in these patients results in false negative cases [7]. Experimental studies have shown that IAH increases dynamic variables [6] and, accordingly, $25 \%$ of patients with IAH may be non-responsive to volume infusion despite a PPV $>12 \%$ [7]. Recently, 
Renner and colleagues [8] found in a porcine model that PPV, but not SVV (derived from pulse contour analysis), remained a sensitive and specific predictor of fluid responsiveness, although the threshold value for PPV increased from $11.5 \%$ (mean IAP $=7 \mathrm{mmHg}$ ) up to $\geq 20.5 \%$ (mean IAP $=26 \mathrm{mmHg}$ ).

Thus, what does the study by Jacques and colleagues [1] add to previous knowledge? First, they confirm former results with a protocol that elegantly combines, for the first time, changes in IAP, blood withdrawal, and fluid loading. Second, they show that SVV, when directly measured using an ultrasound transit-time flow probe placed around the aortic root, is also predictive of fluid responsiveness in IAH. This contrasts with the results reported by Renner and colleagues [8], who acquired SVV via pulse contour monitoring, suggesting that the accuracy of the latter may be altered during IAH. Third, their data strongly suggest that, at least in their experimental conditions, the 'non-responsive' part of the increase in dynamic indices during IAH is due to an increase in right ventricular afterload.

These new data should, however, be extrapolated to critically ill patients with caution. In this study, baseline PPV and SVV were much higher than in humans or in other experimental studies. High tidal volume as used in the study $(13 \pm 1 \mathrm{ml} / \mathrm{kg}$ in the presence of severe reduction in chest wall compliance) may explain, in part, these findings as well as the significant increase in right ventricular afterload, a result not found by Renner and colleagues [8]. In addition, the high IAP level used in the study $(30 \mathrm{mmHg}$ ) corresponds to the more severe level (grade IV, IAH $>25 \mathrm{mmHg}$ [5]) of IAH in patients. Finally, a high but discriminative threshold could be identified by the authors because a precise and controlled value of IAP was induced in all animals, which would not be the case in clinical practice. Thus, what is demonstrated in this study is qualitative (there is an increase in dynamic indices in the presence of IAP, with one part responsive to fluid infusion) rather than quantitative. The threshold values reported by Jacques and colleagues have no direct clinical relevance. The consequences for clinical practice are thus that IAP must be measured in critically ill patients, and dynamic indices such as PPV should be used, but, in order to avoid excess of fluids, higher than classical (10 to 13\%) thresholds should be considered when IAP is increased. As a gradual increase of threshold values with IAP is very likely, no precise value can be recommended so far. In a given patient with increased PPV, a fluid challenge may be performed and the resulting change in PPV/SVV quantified since only the 'preload-dependent part' of PPV is likely to be reduced with volume infusion. Whether the PPV value obtained at completion of such fluid load in such a patient may be used as the 'operational' threshold for the following hours of management, provided that other major determinants of PPV (IAP and tidal volume) are kept constant, can be reasonably hypothesized but deserves further validation.

\section{Abbreviations}

IAH, intra-abdominal hypertension; IAP, intra-abdominal pressure; PPV, respiratory variation in arterial pulse pressure; SVV, respiratory variation in stroke volume.

\section{Competing interests}

The authors declare that they have no competing interests.

Published: 18 March 2011

\section{References}

1. Jacques D, Bendjelid K, Duperret S, Colling J, Piriou V, Viale JP: Pulse pressure variation and stroke volume variation during increased intra-abdominal pressure: an experimental study. Crit Care 2011, 15:R33.

2. Marik PE, Cavallazzi R, Vasu T, Hirani A: Dynamic changes in arterial waveform derived variables and fluid responsiveness in mechanically ventilated patients: A systematic review of the literature. Crit Care Med 2009, 37:2642-2647.

3. Szold A, Pizov R, Segal E, Perel A: The effect of tidal volume and intravascular volume state on systolic pressure variation in ventilated dogs. Intensive Care Med 1989,15:368-371.

4. Biais M, Bernard O, Ha JC, Degryse C, Sztark F: Abilities of pulse pressure variations and stroke volume variations to predict fluid responsiveness in prone position during scoliosis surgery. Br J Anaesth 2010, 104:407-413.

5. Malbrain M: Abdominal compartment syndrome. F1000 Med Rep 2009, 1:86.

6. Malbrain ML, De Laet I: Functional hemodynamics and increased intraabdominal pressure: Same thresholds for different conditions...? Crit Care Med 2009, 37:781-783.

7. Mahjoub Y, Touzeau J, Airapetian N, Lorne E, Hijazi M, Zogheib E, Tinturier F, Slama M, Dupont $\mathrm{H}$ : The passive leg-raising maneuver cannot accurately predict fluid responsiveness in patients with intra-abdominal hypertension. Crit Care Med 2010, 38:1824-1829.

8. Renner J, Gruenewald M, Quaden R, Hanss R, Meybohm P, Steinfath M, Scholz J, Bein B: Influence of increased intra-abdominal pressure on fluid responsiveness predicted by pulse pressure variation and stroke volume variation in a porcine model. Crit Care Med 2009, 37:650-658.

doi:10.1186/cc10074

Cite this article as: Tavernier B, Robin E: Assessment of fluid responsiveness during increased intra-abdominal pressure: keep the indices, but change the thresholds. Critical Care 2011, 15:134. 